\title{
Challenges Facing Children Born with HIV: A Case Study of One Rural Schoolin Oshikoto Region of Namibia
}

\author{
Emilia Ndapandula Mbongo ${ }^{1,}$, Anna Niitembu Hako ${ }^{2}$ \\ ${ }^{1}$ Department of Educational Psychology and Inclusive Education, University Namibia, Rundu, Namibia \\ ${ }^{2}$ Department of Educational Psychology and Inclusive Education, University Namibia, Ongwediva, Namibia \\ Email address: \\ embongo@unam.na (E. N. Mbongo),ahako@unam.na(A. N. Hako) \\ ${ }^{*}$ Corresponding author
}

\section{To cite this article:}

Emilia Ndapandula Mbongo, Anna Niitembu Hako. Challenges Facing Children Born with HIV: A Case Study of One Rural Schoolin Oshikoto Region of Namibia. International Journal of HIVIAIDS Prevention, Education and Behavioural Science.

Vol. 5, No. 1, 2019, pp. 76-81. doi: 10.11648/j.ijhpebs.20190501.20

Received: May 7, 2019; Accepted: June 11, 2019; Published: June 24, 2019

\begin{abstract}
An increasing number of children born with the human immunodeficiency virus (HIV) are surviving well into adolescence and adulthood. This is a direct result of the roll-out of antiretroviral therapy to those infected. This study investigated the challenges facing children born with HIV in one rural school in the Oshikoto region of Namibia. The sample of the study constituted six learners who were purposively selected. Semi-structured interviews and a questionnaire were used to collect data. The results reveal that children know their HIV status and were mostly informed of their status by their caregivers. All the children are on antiretroviral treatment in line with the World Health Organisation (WHO) antiretroviral therapy guidelines, that recommends the initiation of treatment regardless of CD4 count or clinical stage. The results further showed that adherence to treatment remains a problem exacerbated by the secrecy around the virus. Children reported HIV related stigma and discrimination at the hands of adults or peers. Some participants reported feelings of hopelessness, anxiety, isolation and depression. The children's education is also negatively affected by the HIV -positive status. As with other vulnerable children, the HIV-infected child faces many barriers to learning. The study recommends a comprehensive HIV education programme for all Namibians to mitigate the effects that come with misconceptions and a lack of understanding regarding the virus.
\end{abstract}

Keywords: HIV/AIDS, Children, Psycho-Social, Education

\section{Introduction}

The word child refers to a person from the time they were born until they are about 14 years old or marks a period of transition from infancy to childhood [1]. The government of the Republic of Namibia, in the Child Care and Protection Act, however, defines a child as a person who has not yet attained the age of 18 [2]. Children today find it difficult to transition into adults because of the many challenges they face in their daily lives. The situation in many developing countries, including Namibia, is made even worse by the human immunodeficiency virus (HIV), which leads to acquired immunodeficiency syndrome (AIDS).

Globally, it is estimated that more than 35 million people live with HIV [3]. Regrettably, 3.2 million of those who are infected with HIV are under the age of 15. It is further documented that more than two-thirds $(70 \%)$ of all people living with HIV, 24.7 million, live in sub-Saharan Africa; including $91 \%$ of the world's HIV-positive children [3]. Namibia is one of the countries with the highest HIV prevalence in the world at $17.2 \%$, according to the latest surveillance report of the 2016 National HIV Sentinel Survey [4]. Reports further indicate that in 2010/2011, it was estimated that approximately 14,000 children between 0-14 years were infected with HIV of which the majority (estimated at more than 90\%) will have been infected through Mother-to-ChildTransmission (MTCT) [3]. This figure is inconvenient to the country as children are the backbone of each nation's social, economic and political advancement. To mitigate the transmission of HIV from mother to child, a programme called Prevention of the Mother to Child Transmission (PMTCT) was introduced in state hospitals in Namibia in 2003 [5]. It is 
estimated that without interventions, about $20 \%$ to $45 \%$ of babies born to HIV-positive mothers become infected with HIV. As a result, many babies born before the introduction of the PMTCT were born with HIV [3]. The roll-out of the antiretroviral therapy has seen many babies who were born with HIV survive well into adulthood.

Many of these children struggle to cope with the challenges that are a direct result of their positive HIV status. When issues of HIV-positive children are discussed, people rarely distinguish those who are perinatally-infected from those who behaviourally acquired HIV, choosing instead to broadly discuss HIV-positive children as a single population. Studies demonstrate that perinatally-infected children inherit a wide range of biological, social and psychological difficulties [6-9]. Mofenson and Cotton noted that children born with HIVinitiate therapy during a period of rapid growth and face decades, if not a lifetime, of antiretroviral drug exposure [7]. They discussed the side effects of the drugs and diseases that are a direct result of the drugs, such as renal failure. Zanoni discusses that frequent disruptions of social, occupational and academic activities due to clinic appointments and hospitalisations often lead to feelings of anxiety and depression in perinatally HIV infected children [8]. In addition, these children are likely to have experienced a difficult life that could affect their psychosocial well-being, such as losing one or both parents, changing caregivers, experiencing poverty and facing stigma. They are also likely to be struggling with coming to terms with their HIV status at a time when they do not understand what it means to be HIVpositive, nor the importance of adhering to medication.

It is obvious that children who are born with HIV face many challenges different from those who have behaviourally acquired the virus. In Namibia, efforts in the form of campaigns and prevention programmes were made to educate children about the dangers of contracting HIV, however, fewer, if any, focused on those already infected. Where research is concerned, the few studies that were carried out in Namibia focused more on the medical perspective as opposed to the social and behavioural perspectives. As a result, Namibia lends itself to a call for greater research about children who are born with HIV. The current study focused on the psychosocial and health challenges facing children born with HIV. Although perinatally and behaviourally infected children have many commonalities, there are still substantive differences that need to be critically considered when developing programmes and formulating policies in relation to the challenges facing HIV-infected children in Namibia. This study will enrich literature about Namibian children born with HIV and the challenges they face in life.

\section{Methodology}

\subsection{Participants and Setting}

The sample comprised of six learners drawn from one rural school in the Oshikoto region of Namibia. The sample was obtained through purposive sampling.

\subsection{Instruments}

The participating learners responded to a semi-structured questionnaire and an interview schedule. The questionnaire had two main sections: Demographic characteristics of the participants, including gender and age as well as the psychosocial and health challenges perinatally HIV-infected children face. Bertram and Christiansen believe that semistructured questions allow respondents to write their own opinions or record their own experiences in relation to a particular issue without being constrained by closed-ended responses [10]. On the other hand, interviews allow the collection of more in-depth, detailed and descriptive data from a small number of people. In other words, interviews provide researchers with an opportunity to ask probing and clarifying questions and discuss research participants' understanding in relation to the subject under investigation [11-12].

\subsection{Data Collection Procedures}

Ethical approval was granted from the director of Oshikoto Directorate of Education, Arts and Culture and the authority of the school where the study was conducted. Prior to the interview of children, the researchers sought permission from the parents and from the participants themselves. The process of data gathering was framed within ethical principles of assurance of confidentiality and anonymity of participant responses, voluntary participation, informed consent and permission to withdraw at any stage of the study. The children who completed and signed the consent forms were finally involved in the study.

\subsection{Data Analysis}

Data analysis is defined as the process of ordering and structuring research data [10]. Analysis of data was done using the thematic method by classifying various meaningful and relevant responses in clusters. The researchers have chosen the thematic method to analyse data as it helped them pinpoint, examine and record themes within data sets that are crucial to the description of the phenomena under study.

\section{Results and Discussion}

\subsection{Demographic Characteristics}

As indicated in Table 1, the sample consisted of six learners, two males and four females. Their ages ranged between 10 and 17 years of age and they were in grades 4 to 10. As shown in the table, most of the participants are years behind in their schooling. For example, participant 2 is 16 years old and in Grade 5. Under normal circumstances, someone 16 years old would be in grade 10 . Two learners are total orphans and three have lost one parent, while only one participant has both parents. On the participants' living arrangements, only one participant lives with both of her 
parents. One participant lives with her single mother and three live with their grandparents, while the remaining participant lives with a maternal aunt. As the results show, the impact of the HIV/AIDS pandemic has left many children without parents, having lost one and or both parents, and mostly in the care of grandparents.

Table 1. Participants' demographic variables.

\begin{tabular}{llllll}
\hline \multirow{2}{*}{ Participants } & \multirow{2}{*}{ Gender } & \multirow{2}{*}{ Age } & \multirow{2}{*}{ Grade } & Status & \\
\cline { 6 - 6 } & & & & Mother alive & Father alive \\
\hline Participant 1 & Female & 10 & 4 & No & Yes \\
Participant 2 & Male & 16 & 5 & No & Yes \\
Participant 3 & Female & 17 & 10 & Yes & Yes \\
Participant 4 & Female & 16 & 7 & No & No \\
Participant 5 & Female & 17 & 9 & No & No \\
Participant 6 & Male & 16 & 10 & Yes & No \\
\hline
\end{tabular}

\subsection{Participants' HIV Status Awareness}

All the participating learners knew that they are HIVpositive. Two of them indicated that they have known about their HIV status for less than two years, while one has known about her status for more than 10 years. The remaining three could not remember how long they had known their HIV status. With the exception of one participant who was informed by a nurse, the rest of the participants were informed about their status by their caregivers. Vaz, Eng, Maman, Tshikandu and Behets noted that caregivers' decisions to disclose HIV status are influenced by their concerns around treatment adherence, the eminent onset of sexual activity of adolescents, and their desire to protect their children and to protect others from being infected [13].

Furthermore, all the participating children are on antiretroviral (ARV) treatment. This is mainly because Namibia has adopted the World Health Organisation antiretroviral therapy guidelines, which recommends the initiation of treatment regardless of CD4 count or clinical stage [5]. The same policy applies to children and states that all children younger than 15 years old are medically eligible to start antiretroviral treatment (ART) once HIV infection is confirmed. It is hoped that this will improve the survival rate of people living with HIV/AIDS and decrease HIV transmission to HIV un-infected partners.

\subsection{Health Challenges Experienced by Learners Born with HIV}

The results show that none of the participants experienced difficulties accessing hospitals or clinics for treatment. This is because there are two health centres (a clinic and a health mobile clinic unit) within walking distance for the inhabitants of that village. The health mobile clinic unit travels to rural communities offering a range of services from blood pressure screening to HIV treatment and prenatal care. In the past, many people in rural and remote areas had difficulty accessing these services due to the long distances to hospitals or health centres, and the high cost of travel. In contrast to this finding, a study done by Naanyu, Gensberg, Koech, Akinyi, Kamene, Ndege, Siika, Kimayo and Braitstein found that distance to health facilities is a challenge to returning HIV patients to accessing health care [14].

Furthermore, it was indicated that transport was not a challenge when it came to accessing health centres. As discussed above, there are health centres within walking distance. However, accessing health care centres seems to be a problem is some countries such as Tanzania where a study by Nsimba, Irunde and Comoro reported lack of transport especially in remote areas as an additional challenge to accessing hospitals [15]. Similarly, a study carried out in South Africa on barriers to adherence to ART by Azia, Mukumbang and Van Wyk reported transport expenditures and distance as main obstacles for HIV-positive patients to access health facilities [16]. Namibia has thus done well in increasing access to healthcare facilities through standing and mobile clinics.

The results further revealed that four out of six of the participants sometimes missed taking their medication. That is, they do not take their medication every day as prescribed. Achieving and maintaining a high level of adherence is important for the successful viral suppression by the ART [17]. Most of them quoted forgetting to take their medication as the reason they sometimes missed taking their medication. This is exacerbated by the secrecy around their HIV-positive status. In most cases, it is only the primary caregivers who know about the childrens' HIV-positive status. In the event that a caregiver also forgot or if they are away, as it so often happens in rural areas, the child is left with the responsibility of taking their medication on their own. This could derive from the fact that children typically assume less responsibility than adults for medication-taking and often need support to help them to maintain care and to stick to treatment [1].

It was also reported that adherence to treatment is not affected by lack of food. This was probably because of the setting of the study. Our participants are all from a rural area, where people depend on communal crop farming and food is readily available. In addition, the school has a school feeding programme as part of the Ministry of Education, Arts and Culture's efforts to improve access to education, maintain high school attendance and reduce hunger for learners to learn and thrive [18]. The Namibian school feeding programme transition case study revealed that with strong community participation, the feeding programme increased learners' school attendance and improved general health and concentration levels [18]. This finding is contrary to what was found by Weiser, Tuller, Frongillo, Senkungu, Mukiibi and Bangsberg in Uganda that food insecurity and hunger were common barriers to ARV adherence [19]. Similarly, some studies found malnutrition, poverty, food insecurity and consumption pattern, which is less than three meals per day, as significantly associated with non-adherence to ART $[16,20]$

Participants further indicated that a lack of confidentiality among family members is not a concern as most family members did not know their HIV status. Apart from the primary caregivers, the participants' HIV status is a secret. The parents/caregivers keeping the HIV status of their 
children a secret from other family members could be a way of protecting them from being stigmatised and discriminated against. This similar tactic of concealing HIV status was also reported in a study by Siu, Kennedy and Bakeera-Kitaka, where participants indicated that they did not want to disclose to their family members in order to protect them [21]. In support of parents/primary caregivers' decision, Dapaah and Senah confirmed that the persistent stigma associated with HIV infection makes it vital for the clients and caregivers to devise approaches and secretly use counselling, testing and treatment services [22]. HIV-related stigma and discrimination is a major driver of the HIV and AIDS epidemic in Namibia and elsewhere. A study by Angula, Ncama and Frohlich on the assessment of HIV/AIDS stigma in rural Namibian communities confirmed that despite high levels of knowledge on HIV/AIDS, stigma remains a challenge in Namibia [23].

Most participants reported that health officials maintain confidentiality in handling the patients' HIV status. Participants stated that when they visited health facilities, they were taken to a private room where no entry was permitted to another person until the consultation was completed. In a study by Dapaah and Senah, findings also confirmed that health workers observed confidentiality when helping HIV patients with an understanding that clients preferred to access services in privacy in order to avoid being identified by relatives or acquaintances [22]. This result was further confirmed by participants in a study done by Eyassu, Mothiba and Mbambo-Kekana that most of the respondents indicated that privacy was maintained during consultations [24]. The positive patient experiences with the quality of care and patient satisfaction with the attitudes of health workers are important indicators to adherence to treatment. Several studies have reported non-adherence to treatment linked to unpleasant experiences with clinic staff. For example, a study by Srithanaviboonchai, Chariyalertsak, Nontarak, Assanangkornchai, Kessomboon, Putwatana, Taneepanichskul and Aekplakorn revealed that fear of lack of privacy and confidentiality regarding their HIV status at healthcare facilities could further fuel the HIV epidemic as it reduces HIV testing updates and allows HIV-positive people to remain undiagnosed [25].

Most of the participants indicated that health officials were friendly and helpful when they visited health care centres. However, one participant showed that lack of friendly services affected her in accessing health services. She narrated how nurses shouted at her, especially when she missed follow-ups (collecting her medication). In Namibia, for example, there is a strong belief and negative connotation from the public that nurses' attitudes towards patients are not welcoming. One minister supported this credence during the Nursing Bill debate that over the years, nurses have been increasingly accused of bad attitudes and behaviour. This was further affirmed in a study by Bridges, Cheetham, Bayer and Robbins that in Lesotho, young people reported being afraid to get an HIV test because healthcare workers accused them of being promiscuous [26].
None of the participants reported parental permission to access ART as a hindrance to their treatment. This means that all parents or guardians allowed and encouraged their children to access ART. Literature also revealed that many health facilities require parental permission or notification for minors to receive care; a major deterrent for children seeking testing, counselling and HIV-related health care services. For instance, in Africa, only four of 22 countries that responded to a WHO survey had provisions allowing minors to access HIV testing without parental permission [26]

\subsection{Psycho-Social Challenges}

The results show that five out of the six participants have not experienced discrimination at the hands of their friends and/or classmates. HIV continues to be a stigmatised disease, despite significant advances in care and concerted effort to reduce discrimination, stereotypes and prejudice. It was thus a positive message to note that most participants did not experience discrimination because of their HIV status. This prompted the researchers to probe further and find out whether the participants' friends and classmates knew about their HIV status. It emerged that most participants did not tell their classmates and/or friends about their HIV-positive status. Only one girl indicated that she has told her friend about her HIV status and that her friend was very supportive. She emphasised that the friend has promised that she will not tell anyone about her status. As demonstrated here, secrecy around HIV is still a big problem. It is even more difficult for children and adolescents to disclose their HIV status at a time when they themselves are struggling to come to terms with their status.

There was, however, one learner who reported discrimination at the hands of her friend/classmates. She narrated how one day she was turned away from play. In her words:

"... they said go away, we are not playing with you because you are sick." (P4, 16-year-old girl)

This shows that although minimal, discrimination and stigmatisation among children takes place. This explains why many parents or caregivers of HIV-infected children prefer to keep their HIV status a secret. Cooper, Risley, Drake and Bundy described an HIV-infected child as one who is vulnerable to stigmatisation and isolation within the community of young children [27].

All the participants reported that, for the most part, they did not experience discrimination by family members. Further discussion revealed that for many of the participants, only a few people in their households knew about their HIV status. Usually, it is only the immediate caretaker/s, for instance, mother, aunt or grandmother, or any other adult in the family, who know about their HIV status. Secrecy around HIV and AIDS remains a problem because of the stigma that comes with a positive HIV status. One of the reasons for stigma is inadequate information regarding the spread and treatment of HIV. Stigma may also be caused by fear. It is thus important that people are educated about HIV/AIDS. As discussed by Cooper, Risley, Drake and Bundy, literacy is of vital importance in the battle against HIV/AIDS stigma 
annihilation [27].

Only one participant reportedly experienced discrimination at the hands of an uncle. In his own words, his uncle repeatedly says to him: "You are always going to hospitals", "when will you stop going to hospitals?", "when will you get better?" He narrated that this makes him feel very bad and he wished his uncle would stop saying that. It is likely that the uncle does not know that the child is HIV-positive, thus the frequent trips to the clinic.

Participants were also asked whether they worried about other people knowing about their HIV status. Two participants indicated that they were not concerned. The remaining four participants raised their concerns about people knowing their status. They worried about how other people will look at them, what they will say, and how theywill be treated. These questions were especially coming from older children. Younger participants were more concerned about friends not wanting to play with them if they knew they were HIV-positive. This takes us back to the stigma attached to HIV/AIDS and disclosure. Even among children and adolescents, they are already concerned about other people's reactions to their status. These findings are consistent with those by Surkan, et al. who reported that HIV-related stigma and discrimination against children affected by HIV/AIDS was prevalent [6]. Similarly, Dapaah and Senah established that HIV-positive children are faced with problems of stigma and discrimination [22]. The fear of stigma and discrimination lead to reluctance and anxiety to disclose their HIV-positive status.

Two participants reported having thought of committing suicide. Feelings of hopelessness, anxiety, isolation and depression are common among HIV-infected children. Similar results were found by Lawan, Amole, Jahun and Abute where participants reported being anxious or depressed most of the time, commonly from fear of deathand discrimination [28]. HIV infection increases the risk of psychological distress among children and adolescents. It was thus a positive message to learn that the majority of the participants did not report having had suicidal thoughts.

HIV infection also negatively affects children's education. Participants indicated that they sometimes found it difficult to concentrate in class because of their HIV status. As with other vulnerable children, the HIV-infected child faces many barriers to learning. One participant narrated how he sometimes questioned the purpose of education when he could get sick at any time. Some participants felt being absent from school often when they had to go to clinics or hospitals for medical attention or to collect their medication negatively affected their school work. The results in table 1 also indicate that the majority of the participants are behind in their schooling. It thus goes without saying that HIVinfected children face a struggle when it comes to their schooling. It is important that these children have a positive outlook on life, which can be fostered through support groups or youth clubs at school and in the general community. This, however, begs the question whether schools are equipped to handle or support HIV-infected children to ensure their success in school.

\section{Conclusion}

The study revealed noteworthy findings that all participants knew their HIV status, are all on antiretroviral treatment, did not experience transport problems in accessing health care centres and most have not had bad experiences with health care providers. It was however disheartening to learn that some participants experienced stigmatisation at the hands of family members and friends because of their positive HIV status. Adherence to treatment remains a problem for most participants. The participants' schooling is also affected by their HIV status because of the absences from school due their HIV infection. The results have implications for future interventions to support children born with HIV. There is thus a need for continued efforts to sensitize caregivers and the general community on issues affecting perinatally $\mathrm{HIV}$-infected children and the support they need. There is also a need to tackle negative attitudes towards perinatally HIV-infected children both at home and school. To ensure academic success for these children, there is a need for schools to have activities aimed at building their selfesteem and create a sense and hope for the future. It is thus recommended to undertake a comprehensive HIV/AIDS education programme to ensure greater understanding and support of children born with HIV.

\section{Acknowledgements}

We wish to thank Erastus Negonga, a teacher counsellor at Onankali South Combined School in the Oshikoto region of Namibia, for his support in carrying out this project. We also wish to thank the study participants for sharing their experiences with us. Lastly, we thank Cindy van Wyk for language editing and proof-reading.

\section{References}

[1] World Health Organization. (2013). Adolescents falling through gaps in HIV services. WHO: Geneva.

[2] Government Gazette of the Republic of Namibia. (2015). Child Care and Protection Act, 2015. Namibia: Windhoek.

[3] UNICEF (2011). Children and HIV \& AIDS in Namibia. Retrieved from: www.unicef.org/namibia/UNICEF_Nam_2011_Children_and _Aids_Namibia.pdf

[4] Ministry of Health and Social Services (MoHSS). (2016). National guidelines for antiretroviral therapy. Windhoek: MoHSS.

[5] Ministry of Health and Social Services (MoHSS). (2014). National guidelines for antiretroviral therapy $\left(4^{\text {th }}\right.$ ed.). Windhoek: MoHSS. 
[6] Surkan, P. J., Mukheriee, J. S., Williams D. R. \& Eustache E. (2010). Perceived stigma toward children affected by HIV/AIDS and their HIV-positive caregivers in central Haiti. AIDS Care, 22 (11), 803-815. doi: 10.1080/09540120903443392.

[7] Mofenson, L. M. \& Cotton, M. F. (2013). The challenges of success: adolescents with perinatal HIV infection. Journal of the International AIDS society, 16 (1). doi: 10.7448/IAS.16.1.18650.

[8] Zanoni, B. C. (2013). Behavioural, psychiatric and cognitive problems in adolescents with perinatal HIV infection: Unrecognised consequences. HIV Nursing Matters, 4 (1), 14 17.

[9] Vranda, M. N., \&Mothi, S. N. (2013). Psychosocial issues of children infected with HIV/AIDS. Indian J Pshychol Med, 35 (1), 19-22. doi: 10.4103/0253-7176.112195.

[10] Bertram, C., \& Christiansen, I. (2014). Understanding research. An introduction to reading research. Pretoria: Van Schaik Publishers.

[11] Merriam, S. B. (1998). Qualitative research and case study applications in Education. Francisco: Jossy-Bass Publishers.

[12] Du Plooy-Cilliers, F., Davis, C. \& Bezuidenhout, R. M. (2015). Research Matters. Cape Town: Juta\& Company.

[13] Vaz, L. M. E., Eng, E., Maman, E., Tshikandu, T, T. \&Behets, T. (2010). Telling children they have HIV: Lessons learned from findings of a qualitative study in Sub-Sahara Africa. AIDS Patient Care Stds, 24 (4), 247-256. doi: 10.1089/apc.2009.0217.

[14] Naanyu, W. J., Gensberg, B., Koech, B., Akinyi, J., Kamene, R., Ndege, S., Siika, A. M., Kimayo, S. \& Braitstein, P. (2014). Health facility barriers to HIV linkage and retention in Western Kenya. BMC Health Serv Res, 19 (14), 646.doi: 10.1186/s12913-014-0646-6.

[15] Nsimba, S. E. D., Irunde, H. \& Comoro, C. (2010). Barriers to ARV Adherence among HIV/AIDS Positive Persons taking Anti-retroviral Therapy in Two Tanzanian Regions 8-12 Months after Program Initiation. Journal of AIDS \& Clinical Research, 1 (3). Doi: 10.4172/2155-6113.1000111.

[16] Azia, I. N., Mukumbang, F. C. \& VanWyk, B. (2016). Barriers to adherence to antiretroviral treatment in a regional hospital in Vredenburg, Western Cape, South Africa. Southern African Journal of HIV Medicine, $17.41-8$. 10.4102/sajhivmed.v17i1.476.

[17] Van Dyk, A. (2013). HIV and AIDS education, care and counselling: A multidisciplinary approach. Cape Town: Pearson.

[18] Ministry of Education (2012). The Namibian school feeding programme transition case study. Windhoek: Ministry of Education.
[19] Weiser, S. D., Tuller, D. M., Frongillo, E. A., Senkungu, J Mukiibi, N. \& Bangsberg, D. R. (2010) Food insecurity as a barrier to sustained antiretroviral therapy adherence in Uganda. US National Library of Medicine, 5 (4). https://doi.org/10.1371/journal.pone.0010340

[20] Negassie, B., Desalegn, T. \& Mekuriaw, A. (2013). Effects on Nutritional factors on Adherence to Antiretroviral Therapy among HIV-infected adults: a case control study in Northern Ethiopia. BMC Infectious Diseases, 13, 233. https://doi.org/10.1186/1471-2334-13-233

[21] Siu, G. E., Kennedy, C. E. \& Bakeera-Kitaka, S. (2016). Lived experiences, perceptions and treatment disclosure by HIV infected adolescents at the Transition Clinic of Mulago Hospital, Kampala. Eighteenth International AIDS Conference, Vienna, abstract TUAX0103, 2010.

[22] Dapaah, J. M \&Senah, K. A. (2016). HIV/AIDS and Confidentiality; the case of two health centres in Ashanti Region of Ghana. BMC Med Ethics, 17 (1), 41. doi: 10.1186/s12910-016-0123-

[23] Angula, P., Ncama, B. P. \& Frohlich, F. (2015). Assessment of HIV/AIDS stigma in a rural Namibian community. World Journal of AIDS, 5, 199-207. doi: 10.4236/wja.2015.53023

[24] Eyassu, M. A., Mothiba, T. M., \& Mbambo-Kekana, N. P. (2016). Adherence to antiretroviral therapy among HIV and AIDS patients at the Kwa-Thema clinic in Gauteng Province, South Africa. African Journal of Primary Health Care \& Family Medicine, 8 (2). doi: https://dx.doi.org/10.4102\%2Fphcfm.v8i2.924

[25] Srithanaviboonchai, K., Chariyalertsak, S., Nontarak, J., Assanangkornchai, S., Kessomboon, P., Putwatana, P., Taneepanichskul, S. \& Aekplakorn, W. (2017). Stigmatizing attitudes toward people living with HIV among general adult Thai population: Results from the $5^{\text {th }}$ Thai National Health Examination Survey (NHES). https://doi.org/10.1371/journal.pone.0187231

[26] Bridges, E., Cheetham, N., Bayer, L., \& Robbins, S. (2011). Young People Living with HIV around the World: Challenges to health and well-being persist. Washington D. C. $\mathrm{http} / / \mathrm{www}$.advocates for youth.org/storage/advfy/young2.pdf. July 2018.

[27] Cooper, E. S, Risley, C. L., Drake, L. J. \& Bundy, D. A. P. (2007). HIV as part of the life of children and youth, as life expectancy increases: Implications for education. Journal of International Cooperation in Education, 10 (1), 101-113.

[28] Lawan, U. M., Amole, G. T., Jahun, M. G. \& Abute, J. E (2015). Psychosocial challenges and adherence to antiretroviral therapy among HIV-positive adolescents attending an ART center in Kano, northwestern Nigeria. Int $J$ Med Sci Public Health, 4 (10): 1439-1444. doi: 10.5455/ijmsph.2015.27042015297. 